\title{
Mobile App and Digital System for Patients After Myocardial Infarction (AfterAMI); Study Protocol for a Randomized Controlled Trial
}

\section{Bartosz Krzowski}

Medical University of Warsaw: Warszawski Uniwersytet Medyczny

Michał Peller ( $\sim$ michalpeller@gmail.com )

Medical University of Warsaw: Warszawski Uniwersytet Medyczny

Maria Boszko

Medical University of Warsaw: Warszawski Uniwersytet Medyczny

Paulina Hoffman

Medical University of Warsaw: Warszawski Uniwersytet Medyczny

Natalia Żurawska

Medical University of Warsaw: Warszawski Uniwersytet Medyczny

Karolina Jaruga

Medical University of Warsaw: Warszawski Uniwersytet Medyczny

Kamila Skoczylas

Medical University of Warsaw: Warszawski Uniwersytet Medyczny

\section{Gabriela Osak}

Medical University of Warsaw: Warszawski Uniwersytet Medyczny

Łukasz Kołtowski

Medical University of Warsaw: Warszawski Uniwersytet Medyczny

Marcin Grabowski

Medical University of Warsaw: Warszawski Uniwersytet Medyczny

Grzegorz Opolski

Medical University of Warsaw: Warszawski Uniwersytet Medyczny

Paweł Balsam

Medical University of Warsaw: Warszawski Uniwersytet Medyczny

\section{Research Article}

Keywords: Acute myocardial infarction, telemedicine, telehealth, mobile application, cardiac rehabilitation

Posted Date: October 29th, 2021

DOl: https://doi.org/10.21203/rs.3.rs-517597/v2 
License: (c) (i) This work is licensed under a Creative Commons Attribution 4.0 International License. Read Full License 


\section{Abstract}

Background: Treatment of acute myocardial infarction has been studied and improved over the past years. However, the initial months after myocardial infarction are crucial from the perspective of the patient's prognosis. It is extremely important to take care of all cardiovascular risk factors.

Mobile application 'afterAMI' supported by a web system is a novel telemedical tool developed to support patients and physicians during cardiac rehabilitation. The application has an educational model with a focus on cardiovascular risk factors and lifestyle after myocardial infarction. Moreover, it offers a module that controls vital signs like blood pressure, heart rate, weight, and many others. Additionally, the application will send reminders for better drug adherence.

Methods: A group of 100 patients with myocardial infarction on admission at the $1^{\text {st }}$ Chair and Department and of Cardiology, Medical University of Warsaw, will be recruited into the study. The project aims to assess the impact of the application-supported model of care in comparison with standard rehabilitation. At the end of the study, cardiovascular risk factors will be analysed, along with issues like rehospitalizations, patients' knowledge of risk factors, returning to work, and quality of life. In this prospective, open-label, randomized, single-center study, all 100 patients will be observed for 6 months after discharge from the hospital. Endpoints will be assessed during control visits 1-and 6-months after inclusion into the study.

Discussion: This project is an example of a telemedical solution application embracing everyday clinical practices, conforming with multiple international cardiac societies' guidelines. Cardiac rehabilitation process enhancements are required to improve patients' prognosis. The evidence regarding the use of the mobile application in the described group of patients is limited and usually covers a small number of participants. The described study aims to discuss whether telemedicine use in this context is beneficial for the patients.

Trial registration: ClinicalTrials.gov, NCT04793425, registered 11 March 2021.

\section{Introduction}

\section{Background and rationale $\{6 a\}$}

Cardiovascular diseases are the leading cause of death and a focal contributor to disability. Acute myocardial infarction (AMI) treatment has improved significantly over the past years, and the vast majority of patients with the acute coronary syndrome (ACS) can be treated in the cath lab. The mortality rate following AMI varies between countries, but an overall decrease has been observed (1). Nevertheless, $12 \%$ of the patients die within one year after AMI (2). Therefore, efforts should be made to optimize the cardiac rehabilitation process. It is crucial to focus on preventing future ischemic events by providing optimal care for patients at-risk (3). Secondary prevention aims to control all cardiovascular disease (CVD) risk factors, which may be challenging in everyday practice. Jankowski et al. reported that only 
$2.9 \%$ of patients with coronary artery disease (CAD) have all CVD risk factors controlled corresponding to values recommended in the guidelines (4). Proper CVD risk factor control remains a challenge in the realworld setting.

Several efforts are being made to improve patients' prognosis. The latest approach to improve cardiac rehabilitation is the use of novel telehealth-based solutions. Over 3.2 billion smartphones are used globally, and the mobile applications market is expected to grow by $18.4 \%$ between 2018 and 2026 . Therefore, enhancing cardiac rehabilitation by mobile application support appears to be a promising tool. Telemedicine has proved to be an effective tool in many clinical scenarios. Widmer et al. demonstrated that augmentation of usual cardiac rehabilitation with an online and smartphone-based program improved CVD risk factor management and reduced rehospitalizations or emergency department visits by $40 \%(p<0.05)(5)$. Naturally, not all cardiac patients are capable of using smartphones. Gallagher et al. reported $54.6 \%$ of cardiac patients eligible for attending cardiac rehabilitation used technology for health purposes. Patients used it to access information on health conditions and medications mainly(6). Coorey et al. concluded in the meta-analysis that mobile applications have a beneficial influence on CVD risk factors control, but more scientific evidence is required to enhance the implementation of telemedicine into clinical practice (7).

Although several international cardiac societies recommend telemedicine use $(8,9)$, evidence-based conclusions are required to adjust specific telemedical tools individually to the patient and improve the prognosis. The influence of mobile application support on cardiac rehabilitation in a European setting is yet to be studied.

\section{Objectives $\{7\}$}

This study will aim to determine the effect of mobile application-supported cardiac rehabilitation on CVD risk factors control, rehospitalization, and emergency department visits, quality of life, and the ability to return to work. We hypothesized that cardiac rehabilitation enhancement with the mobile application would improve prognosis by CVD risk factors management. Moreover, we expect that the intervention will improve patients' quality of life.

\section{Trial design $\{8\}$}

This protocol is a randomized, open-label, superiority, interventional study with two arms. Participants will be randomized to (1) a control group (CG) with standard cardiological care or (2) a mobile applicationsupported interventional group (IG). The anticipated number of participants is 100 .

\section{Methods: Participants, Interventions And Outcomes}

\section{Study setting $\{9\}$}


This single-center study will be carried out at the $1^{\text {st }}$ Department of Cardiology at the Medical University of Warsaw, an academic, public hospital in the capital of Poland. Cardiologists and fellows of cardiology will conduct all study-related procedures. The Department ensures all treatment options for patients with $\mathrm{AMI}$ and during their cardiac rehabilitation process. It is regarded as the leading Department of Cardiology in Poland.

\section{Eligibility criteria $\{10\}$}

\section{Inclusion Criteria:}

- signing the informed consent to participate in the study

- hospitalization due to myocardial infarction, based on the Guidelines on Fourth Universal Definition of Myocardial Infarction (10)

- owning a mobile device with Internet access and the Android/iOS operating system

- age $\geqslant 18$ years old

- positive results of a test verifying the basic skills of using mobile applications

\section{Exclusion Criteria:}

- Life expectancy shorter than 6 months due to non-cardiac illness

\section{Who will take informed consent? $\{26 a\}$}

After screening a list of patients admitted to the department of cardiology, a staff member will check the reason for admission. A study team member will approach every patient presenting with $\mathrm{AMI}$, and the inclusion criteria will be assessed. The study design will be thoroughly described to the patient, including all potential benefits, harms, and ethical implications. Each eligible patient will be proposed to enter the study. If the patient agrees to participate in the research, the informed consent will be signed in 2 copies: one for the participant and one for the research archives. Every participant will receive a note with a summary of the study design.

\section{Additional consent provisions for collection and use of participant data and biological specimens $\{26 \mathrm{~b}\}$}

Not applicable; this trial does not have biological specimens.

\section{Interventions}

\section{Explanation for the choice of comparators $\{6 b\}$}

Participants will be randomly assigned to one of the two groups (1) a control group with standard cardiological care or (2) a mobile application-supported interventional group. The trial is set in a relatively big, academic centre. Every year approximately 400 patients is hospitalised due to acute myocardial infarction. However, a significant part of those patients is unable to use mobile application and therefore 
they don't meet one of the main inclusion criterion. Going further, only some of the patients who meet inclusion criteria are willing to take part in a trial. The recruitment began in December 2020.

Approximately 7 patients per month is included into the trial. Last patient recruitment is expected in Q1 2022.

\section{Intervention description $\{11 \mathrm{a}\}$}

The rehabilitation process of patients in the intervention group will be supported by the mobile application (afterAMI) with a synchronized, dedicated web page.

Patients in the intervention group will also be given access to educational data regarding their diseases. Every educational chapter was prepared by a cardiologist experienced in managing patients after MI.

Additionally, every patient will regularly receive messages with notifications about lifestyle interventions and adherence to therapy. An optimal scheme for messeges quantity and structure has not bec described yet in cardiac patients. In recent metaanalysis Bashi et al. concluded that the results of mobile application assisted patient education generally shows positive result. However due to poor reporting quality and heterogenity of the interventions further studies are needed to develop optimal educational scheme (11). On the other hand a cardiac telerehabilitation programme augmented by short message service (SMS) deliverd to patients once a week showed improvement in physical fitness and quality of life (12). Based on those reports authors of this trial decided to send 2 messages weekly.

Another essential feature of the application is the possibility to report patients' vital signs (blood pressure, heart rate, weight, and other), which will be daily analyzed and if necessary, a short message will be sent to the patient, advising to present to the primary healthcare clinic or emergency department. A possible clinical scenario is an alarming rapid bodyweight increase, which might foreshadow incoming heart failure exacerbation.. Another potential use of the application lies when the patients report rapid pulse. In this case, new-onset atrial fibrillation might be suspected. In every case requiring confirmation, the patient will be referred to the nearest emergency room. However, all patients will be informed that they should immediately present to the nearest emergency unit or contact the emergency services in case of recurring angina or any other acute complaints.

Additionally, the application will send notifications with reminders to take drugs. This solution has been previously evaluated in many studies and proved to be a successful tool in increasing adherence to the therapy (13).

Moreover, the application includes a module with air pollution parameters measured amidst the localization setup. If they exceed the alarming levels, the patients will be notified, and outdoor physical activities will be minimized.

Moreover, every patient will have a medical history card created based on the discharge documents from the hospital. This solution aims for the patient always to have brief information about underwent 
coronary interventions. This knowledge might be crucial for the physicians performing the subsequent percutaneous coronary intervention (PCl) and might decrease time-to-balloon.

Finally, the application offers a possibility to text message and call the cardiologists and the hospital. We believe that this will translate into better work organization, better time management (as fewer consultations are likely to be missed by patients), and patient safety. All patients randomised to IG will be thoroughly trained in application features and capabilities before discharge from the hospital.

In contrary, patients randomised to the CG will be provided with the best available care worked out based on the available literature (14). Rehabilitation programmes are voluntary. They consist of regular cardiac consultations with experienced physician, psychological sessions, exercise trainings, stress management programmes and diatary recommendations. Patient is offered a support for a year after MI. Patients who refuse to take part in the rehbilitation programme are taken care of by general practitioner. Thanks to randomisation patients distribution is expected to be close to even.

All data impleneted into the application was prepared by experienced cardiologists with big experience in both clinical practice and eHealth use on a daily basis.

There are several mobile applications offering simple feature for general population (i.e. blood pressure diaries). However, according to autorhs' knowledge this is one of the very first, which combines several options previously tested in clinical settings, which proved to be effective, Moreover mobile applications dedicated for patients after $\mathrm{Ml}$ are lacking, and one should keep in mind how unstable first weeks and months after discharge from hospital can be. Described tool should be regarded as a possibility of impoving patients' prognosis when added on top of the current practice.

\section{Criteria for discontinuing or modifying allocated interventions $\{11 \mathrm{~b}\}$}

The only criterion for discontinuing is the participants' request.

\section{Strategies to improve adherence to interventions $\{11 \mathrm{c}\}$}

Not applicable; this trial does not have strategies to improve adherence.

\section{Relevant concomitant care permitted or prohibited during the trial $\{11 \mathrm{~d}\}$}

Not applicable; this trial does not have concomitant care permitted or prohibited.

\section{Provisions for post-trial care $\{30\}$}

Not applicable; participants will continue with traditional care after the trial is finished. Trial participation is beneficial for the patients due to more intense medical care. Every patient included in the study will have two additional cardiological consultations.

\section{Outcomes $\{12\}$}


All 100 patients will be observed for 6 months after discharge from the hospital. We will assess the Endpoints twice, during two control visits, in 1-and 6-months, after inclusion in the study. Primary outcomes encompass rehospitalization and urgent outpatient visit combined and second combined Endpoint; cardiovascular risk factors control (blood pressure, body mass, nicotinism, dyslipidemia). Detailed target values regarding risk factors control have been presented in Table 1 based on ESC Chronic Coronary Syndrome guidelines (15). Secondary outcomes will include quality of life and depression severity assessment (MacNew, EQ-5D-5L, and DASS-21 questionnaires), cardiovascular risk factors' knowledge, and return to work in case of professionally active patients.

Moreover, data collection will include sex, age, laboratory tests, and prescribed pharmacotherapy.

\section{Rehospitalization or urgent outpatient visit}

The number of admissions to hospital or urgent outpatient visits will be assessed between baseline and control visits.

\section{Cardiovascular risk factors}

\section{Blood pressure}

All patients at discharge from the hospital are asked to measure and note blood pressure values daily. The mobile application-obtained mean blood pressure values covering 5 days prior to their visits will be averaged for the IG patients. The mean blood pressure values of CG-comprised patients will also be averaged but based only on the presented notes. Additionally, meeting guidelines-based recommended values will be checked in both groups. Hypertension is one of the main cardiovascular risk factors, with a significant prevalence of 1.13 billion worldwide (16). Hypertension control in patients after Ml is crucial and correlates with patients' prognosis (17).

\section{Body mass}

All patients will be weighed at admission to the hospital and during control visits 1-and 6-months after discharge. Weight change will be measured. Maintaining healthy body weight is one of the fundamental aspects of preventing cardiovascular diseases and an essential treatment element after an MI.

\section{Nicotinism}

All patients will be asked about smoking at admission and during control visits 1- and 6-months after discharge. Smoking cessation is one of the main goals of patients after MI. Quitting smoking is necessary to reduce the risk of another ischemic incident. It has been documented that patients who stopped smoking have reduced the risk of another MI by $50 \%$ (18).

\section{Dyslipidemia}


All patients after MI will have their cholesterol levels measured during hospitalization. Subsequent cholesterol level measurements will be performed during control visits. According to ESC guidelines, different groups of patients have different LDL cholesterol target values, which should be met during the rehabilitation process (19). Lowering LDL cholesterol levels correlates with a better prognosis after MI (20).

\section{Secondary Outcome Measures:}

\section{Quality of life - MacNew (21)}

Quality of life will be assessed with the MacNew questionnaire containing 27 questions. The scoring of the MacNew is as follows, the maximum score in every domain is 7 [high quality], and the minimum is 1 [poor quality]. The quality of life is assessed in the context of physical, emotional, and social aspects.

Quality of life - descriptive profile of the respondent's health state (22)

Quality of life will be assessed with the help of the EQ-5D-5L questionnaire, gauging 5 aspects: mobility, self-care, usual activity, pain/discomfort, and anxiety/depression. Every segment is assessed based on a 5 level scale:

LEVEL 1: indicating no problem; LEVEL 2: indicating slight problems; LEVEL 3: indicating moderate problems; LEVEL 4: indicating severe problems; LEVEL 5: indicating an inability to/extreme problems

DASS 21 - Depression, anxiety and stress assessment (23)

Depression, anxiety, and stress assessment will be assessed with DASS 21 scale. The DASS is a quantitative measure of distress along the 3 axes of depression, anxiety, and stress.

There are 21 questions; each has 4 possible answers:

0 Did not apply to me at all - NEVER

1. Applied to me to some degree, or some of the time - SOMETIMES

2. Applied to me to a considerable degree, or a good part of the time - OFTEN

3. Applied to me very much, or most of the time - ALMOST ALWAYS

Seven questions are assigned to every aspect: depression, anxiety, and stress.

Higher result in each section contributes to higher severity in depression, anxiety, and stress.

Cardiovascular risk factors knowledge

Cardiovascular risk factors knowledge will be assessed with a previously prepared questionnaire (10 questions). 
Return to work

In the cases of previously working patients, the likelihood of returning to work will be assessed, and the timing of returning to work will be counseled.

\section{Participant timeline $\{13\}$}

Figure 1 shows the recommended SPIRIT figure with the participant timeline.

\section{Sample size $\{14\}$}

Currently, the data regarding the reduction of rehospitalizations or urgent visits impacted by mobile applications is limited. Previous studies were conducted on smaller populations. This calculation was based on Widmer and colleagues' (5) study, considering rehospitalization and urgent ambulatory visits comparing the effects of an online and smartphone-based program with standard rehabilitation on the mentioned endpoint. There was a $40 \%$ decrease in the primary endpoint. $50 \%$ of patients in the control group and $20 \%$ in the interventional arm were rehospitalized or visited the emergency department.

An online calculator (https://clincalc.com/) was used to determine the sample size, assuming the power of $80 \%$ and significance of $5 \%$. A total of 76 patients (38 per group) were required. However, considering a possible lost-to-follow-up group and possible dropouts, a decision to recruit 100 patients was made.

\section{Recruitment \{15\}}

Participant recruitment will occur daily from Monday to Friday. Patients eligible for the study and willing to participate will be provided with complete and detailed information on the study protocol. Lastly, patients will sign two copies of the informed consent.

\section{Assignment of interventions: allocation}

\section{Sequence generation $\{16 \mathrm{a}\}$}

\section{Concealment mechanism \{16b\}}

\section{Implementation $\{16 \mathrm{c}\}$}

Randomization will be performed with an online tool available at https://www.randomizer.org. A hundred sets will be generated, each with a number (1 for CG and 2 for IG). All allocations to CG and IG will be executed before the study begins. The list of subsequent allocations will not be visible for the recruiting physician until the initial eligibility assessment of the patient and obtaining the patient's consent for study participation. After collecting the initial documentation, the physician will be unblinded and receive the group allocation information from the principal investigator. It should be underlined that the person who checks for inclusion criteria and introduces the patient into the trial protocol is blinded until patient agreement. Figure 2 shows the study design flow chart, describing all the steps of the study (Fig. 2). 
Assignment of interventions: Blinding

\section{Who will be blinded $\{17 \mathrm{a}\}$}

This is an open-label study. The only blinded study participants will be physicians who will check the patients' will to participate in the study. After obtaining agreement, the randomized group will be unblinded. Person performing statistical analysis will be blinded, as well as nurses collecting blood samples on the follow-up visits. Taking into consideration use of mobile application in everyday practice it is impossible to blind phisicians performing follow-up visits. It could be regarded as ethically doubtful, because based on the data provided via mobile application clinical decisions an be made (i.e. blood pressure treatment augmentation).

\section{Procedure for unblinding if needed $\{17 \mathrm{~b}\}$}

This is an open-label study. The recruiting physicians will be unblinded after obtaining agreement from the patient.

\section{Data collection and management}

\section{Plans for assessment and collection of outcomes $\{18 \mathrm{a}\}$}

The assessments will be carried out at the $1^{\text {st }}$ Department of Cardiology, Medical University of Warsaw, and the rehabilitation will follow a scheduled program. The intervention involves installing the afterAMI mobile application on patients' smartphones. Control visits will take place in the cardiac ambulatory clinic. Blood samples will be sent to the local laboratory. Patients will be asked to fulfill the MacNew, EQ5D-5L, and DASS21 questionnaires. An experienced cardiologist familiar with the study protocol will conduct all the control visits. Highest data quality is one author's primary goal while conducting a trial. Every information will be entered into database and subsequently checked by another investigator. Every investigator will have valid good clinical practice certificate.

\section{Plans to promote participant retention and complete follow-up $\{18 \mathrm{~b}\}$}

Patients will be called to schedule the control visit's date after discharge. Additionally, patients in the IG will receive a notification in their mobile app reminding them about the upcoming ambulatory visits.

\section{Data management $\{19\}$}

All data collected during the study and medical documents will be protected and stored in a room dedicated to clinical trials' records. All electronic materials will be duly stored in the principal researcher's computer protected with a password known only to the principal researcher. Additionally, a backup in the cloud will be performed after new data collection.

Confidentiality $\{27\}$ 
The highest level of confidentiality will be applied. The participants' data will be kept separately from any identifying information. According to good clinical practice, all investigators will make every effort to keep the sensitive data confidential.

Plans for collection, laboratory evaluation and storage of biological specimens for genetic or molecular analysis in this trial/future use $\{33\}$

Not applicable; all blood samples will be tested in the laboratory according to the locally implemented standards and subsequently utilized. There are no plans for future blood use. No other biological specimen will be tested during this trial.

\section{Statistical methods}

\section{Statistical methods for primary and secondary outcomes $\{20 \mathrm{a}\}$}

The distribution of continuous variables will be estimated using the Shapiro-Wilk test. In the case of variables with a normal and non-normal distribution, the groups will be compared using the Student's ttest and the non-parametric Mann-Whitney $U$ test. The comparison of qualitative variables between the groups will be performed using the Fisher exact test. In order to compare changes in the values of continuous variables over time, the analysis of variance will be performed. To compare the outcome of the patients, the Kaplan-Meier estimators will be utilized. Primary endpoint will be assessed together and separately. For quantitative variables the change from baseline will be assessed. Additionally, in order to diminish differences in sex and age related to group size, propensity score matching analysis will be performed as well.

\section{Interim analyses $\{21 \mathrm{~b}\}$}

Not applicable; interim analyses will not be performed in the present study.

\section{Methods for additional analyses (e.g. subgroup analyses) $\{20 \mathrm{~b}\}$}

Not applicable; additional analyses are not planned in the present study.

\section{Methods in analysis to handle protocol non-adherence and any statistical methods to handle missing data $\{20 \mathrm{c}\}$}

A per-protocol analysis will be performed after completing all of the follow-up visits. In the analysis, we will include all patients who meet inclusion criteria and sign informed consent regardless of the follow-up completion. Statistical calculations will be performed twice; after obtaining data from the first follow-up visit from all patients and after the final follow-up, 6 months after discharge. In case of missing data, patients will be excluded from the particular analysis.

Plans to give access to the full protocol, participant level-data and statistical code $\{31 \mathrm{c}\}$ 
Not applicable; access to the data sets and statistical code are not planned for this study. However, this material might be available upon an adequately justified request to the corresponding author while maintaining participants' anonymity.

\section{Oversight and monitoring}

\section{Composition of the coordinating centre and trial steering committee $\{5 \mathrm{~d}\}$}

The research center is coordinated and managed by GO and the researcher MG. The principal investigator-BK will direct the trial. There is no additional steering committee considered for this study. All researchers will meet monthly to discuss the recruitment progress and solve possible issues.

\section{Composition of the data monitoring committee, its role and reporting structure $\{21 \mathrm{a}\}$}

No additional external monitoring committee is considered for this study. The principal investigator will meet monthly with all the researchers involved in this study via an online platform (Zoom) to discuss the research progress and solve possible issues. Researchers are instructed to immediately report any issues to the principal investigator, who will subsequently organize an additional committee meeting and inform the board review committee from the Medical University of Warsaw and the Ethics Committee of the Medical University of Warsaw, Warsaw, Poland, when appropriate.

\section{Adverse event reporting and harms $\{22\}$}

Serious adverse events of mobile application usage have not been described so far. However, any adverse events will be reported and thoroughly documented and presented in the study summary.

\section{Frequency and plans for auditing trial conduct $\{23\}$}

The principal investigator will continuously monitor the trial conduct. Monthly reports regarding any potential adverse events and protocol violations will be prepared. Additional auditing will be conducted on request from the Ethics Committee of the Medical University of Warsaw.

\section{Plans for communicating important protocol amendments to relevant parties (e.g. trial participants, ethical committees) $\{25\}$}

Any protocol amendments will be reported to and approved by the Ethics Committee of the Medical University of Warsaw. All modifications will be updated at clinicaltrials.gov by the principal investigator (BK). Any important protocol modifications will be communicated to the investigators and patients verbally.

\section{Dissemination plans $\{31$ a $\}$}

The participants will receive a full report with the results of their assessments after the data is analyzed. At the end of the study, the principal investigator will contact the participants to provide them with final 
educational materials and information regarding secondary cardiovascular prevention. Study outcomes will be reported at both local and international cardiological conferences. The final study results will be submitted to a peer-reviewed indexed scientific journal within the 3 years after the last patient's enrollment.

\section{Discussion}

This study aims to investigate the effects of mobile application-assisted cardiac rehabilitation after MI on rehospitalization rate, cardiovascular risk factors control, and patients' quality of life. Considering the alarmingly high percentage of deaths within the first year after $\mathrm{Ml}$, there is a real need for improving the rehabilitation process by intensifying cardiovascular risk factors' control. Standard schemes consisting of pharmacotherapy and lifestyle changes have been thoroughly studied and recommended in the guidelines (3). Novel methods of increasing the patient's involvement and adherence are of the highest importance.

Telemedicine is a rapidly growing branch of the diagnostic, therapeutic, and rehabilitation process. Cardiology is one of the primary beneficiaries of the newly implemented tools. Several cardiac guidelines recommend enhancing everyday clinical practice with mHealth solutions, which seem to prove this assumption $(9,24,25)$. Even though there are various mobile applications available for patients, only a few were validated in clinical settings, focusing on mentioned endpoints. Additionally, many of them were not developed by clinicians or experts in their fields of interest. It is crucial to establish whether such digitally supported rehabilitation may translate into a better prognosis through cardiovascular risk factors control improvement. In previous studies, mobile applications proved to reduce the rehospitalization rate after $\mathrm{Ml}$, but the data were collected only in a smaller sample of patients and different healthcare systems (5). Johntson et al. reported that a simple, dedicated mobile application results in better selfreported drug adherence and may correlate with lifestyle changes and quality of life (26). However, the discussion regarding the use of mobile applications in cardiac patients is still ongoing.

Despite several strengths, this study also has some limitations. Firstly, we will be unable to assess the mortality rate due to a short observation period and small sample size (both due to organizational issues). However, we do not expect this parameter to differ between groups. Additionally, this singlecenter analysis might be biased due to internal protocols, which might differ in other clinics.

Nonetheless, our project stands as a practical example of implementing modern solutions to improve patients' prognoses. If our assumptions regarding the potential beneficial effects of using the afterAMI application appear trustworthy, this study will provide a stronger voice in discussing broader telemedicine usage in everyday clinical practice.

\section{Trial Status}

Recruiting. 
Version 2. August 29th, 2021.

Date recruitment began: December 1, 2020. Approximate date when recruitment will be completed: March $31,2022$.

\section{Abbreviations}

AMI

Acute Myocardial Infarction

ACS

Acute Coronary Syndrome

CVD

Cardiovascular Disease

CAD

Coronary Artery Disease

CG

Control Group

IG

Interventional Group

$\mathrm{PCl}$

Percutaneous Coronary Intervention

\section{Declarations}

\section{Acknowledgements}

We thank all the participants for their involvement in the study.

\section{Authors' contributions $\{31 \mathrm{~b}\}$}

B.K. is the principal investigator. B.K., M.P., P.B., Ł.K. and G.Op. were responsible for the concept and the design of the study. M.B. P.H., K.J., K.S., N. Ż. and G. Os. were involved in data collection. B.K. and M.P, are responsible for statistical analysis. B.K., M.P and P.B. wrote the first version of the manuscript. All authors edited and approved the final version of the manuscript.

\section{Funding $\{4\}$}

The work is carried out in the years 2020 to 2022, financed by the subsidy allocated to science, obtained by the Medical University of Warsaw.

\section{Availability of data and materials $\{29\}$}

After the final study is published, the materials and data will be available upon a reasonable request to the corresponding author. 


\section{Ethics approval and consent to participate $\{24\}$}

The study has been developed in accordance with the declaration of Helsinki guidelines and was approved by the Scientific Ethics Committee of the Medical University of Warsaw (KB 150/2020). All participants will be asked to provide written informed consent before inclusion. Please see the annexing "Ethical Approval."

\section{Consent for publication $\{32\}$}

Please see the annexing "Informed Consent."

\section{Competing interests $\{28\}$}

None declared

\section{References}

1. Degano IR, Salomaa V, Veronesi G, Ferrieres J, Kirchberger I, Laks T, et al. Twenty-five-year trends in myocardial infarction attack and mortality rates, and case-fatality, in six European populations. Heart. 2015;101(17):1413-21.

2. Santos IS, Goulart AC, Brandao RM, Santos RC, Bittencourt MS, Sitnik D, et al. One-year Mortality after an Acute Coronary Event and its Clinical Predictors: The ERICO Study. Arq Bras Cardiol. 2015;105(1):53-64.

3. Authors/Task Force M, Piepoli MF, Hoes AW, Agewall S, Albus C, Brotons C, et al. 2016 European Guidelines on cardiovascular disease prevention in clinical practice: The Sixth Joint Task Force of the European Society of Cardiology and Other Societies on Cardiovascular Disease Prevention in Clinical Practice (constituted by representatives of 10 societies and by invited experts): Developed with the special contribution of the European Association for Cardiovascular Prevention \& Rehabilitation (EACPR). Eur J Prev Cardiol. 2016;23(11):NP1-NP96.

4. Jankowski P, Kosior DA, Sowa P, Szostak-Janiak K, Koziel P, Krzykwa A, et al. Secondary prevention of coronary artery disease in Poland. Results from the POLASPIRE survey. Cardiol J. 2020;27(5):53340.

5. Robert Jay Widmer TA, Lilach Lerman and Amir Lerman. THE AUGMENTATION OF USUAL CARDIAC REHABILITATION WITH AN ONLINE AND SMARTPHONE-BASED PROGRAM IMPROVES CARDIOVASCULAR RISK FACTORS AND REDUCES REHOSPITALIZATIONS. Journal of the American College of Cardiology. 2014;63(12 Supplement).

6. Gallagher R, Roach K, Sadler L, Glinatsis H, Belshaw J, Kirkness A, et al. Mobile Technology Use Across Age Groups in Patients Eligible for Cardiac Rehabilitation: Survey Study. JMIR Mhealth Uhealth. 2017;5(10):e161.

7. Coorey GM, Neubeck L, Mulley J, Redfern J. Effectiveness, acceptability and usefulness of mobile applications for cardiovascular disease self-management: Systematic review with meta-synthesis of 
quantitative and qualitative data. Eur J Prev Cardiol. 2018;25(5):505-21.

8. Piotrowicz R, Grabowski M, Balsam P, Koltowski L, Kozierkiewicz A, Zajdel J, et al. ["Baltic Declaration"-telemedicine and mHealth as support for clinical processes in cardiology. The opinion of the Committee of Informatics and Telemedicine of the Polish Society of Cardiology and Telemedicine Clinical Sciences Committee of the PAS]. Kardiol Pol. 2015;73(7):575-84.

9. Steinberg JS, Varma N, Cygankiewicz I, Aziz P, Balsam P, Baranchuk A, et al. 2017 ISHNE-HRS expert consensus statement on ambulatory ECG and external cardiac monitoring/telemetry. Heart Rhythm. 2017;14(7):e55-e96.

10. Thygesen K, Alpert JS, Jaffe AS, Chaitman BR, Bax JJ, Morrow DA, et al. Fourth Universal Definition of Myocardial Infarction (2018). J Am Coll Cardiol. 2018;72(18):2231-64.

11. Bashi N, Fatehi F, Fallah M, Walters D, Karunanithi M. Self-Management Education Through mHealth: Review of Strategies and Structures. JMIR Mhealth Uhealth. 2018;6(10):e10771.

12. Frederix I, Hansen D, Coninx K, Vandervoort P, Vandijck D, Hens N, et al. Medium-Term Effectiveness of a Comprehensive Internet-Based and Patient-Specific Telerehabilitation Program With Text Messaging Support for Cardiac Patients: Randomized Controlled Trial. J Med Internet Res. 2015;17(7):e185.

13. Marquez Contreras E, Marquez Rivero S, Rodriguez Garcia E, Lopez-Garcia-Ramos L, Carlos Pastoriza Vilas J, Baldonedo Suarez A, et al. Specific hypertension smartphone application to improve medication adherence in hypertension: a cluster-randomized trial. Curr Med Res Opin. 2019;35(1):167-73.

14. Ambrosetti M, Abreu A, Corra U, Davos CH, Hansen D, Frederix I, et al. Secondary prevention through comprehensive cardiovascular rehabilitation: From knowledge to implementation. 2020 update. A position paper from the Secondary Prevention and Rehabilitation Section of the European Association of Preventive Cardiology. Eur J Prev Cardiol. 2020:2047487320913379.

15. Knuuti J, Wijns W, Saraste A, Capodanno D, Barbato E, Funck-Brentano C, et al. 2019 ESC Guidelines for the diagnosis and management of chronic coronary syndromes. Eur Heart J. 2020;41(3):407-77.

16. Collaboration NCDRF. Worldwide trends in blood pressure from 1975 to 2015: a pooled analysis of 1479 population-based measurement studies with 19.1 million participants. Lancet. 2017;389(10064):37-55.

17. Rosendorff C, Lackland DT, Allison M, Aronow WS, Black HR, Blumenthal RS, et al. Treatment of hypertension in patients with coronary artery disease: a scientific statement from the American Heart Association, American College of Cardiology, and American Society of Hypertension. Circulation. 2015;131(19):e435-70.

18. Colivicchi F, Mocini D, Tubaro M, Aiello A, Clavario P, Santini M. Effect of smoking relapse on outcome after acute coronary syndromes. Am J Cardiol. 2011;108(6):804-8.

19. Authors/Task Force M, Guidelines ESCCfP, Societies ESCNC. 2019 ESC/EAS guidelines for the management of dyslipidaemias: Lipid modification to reduce cardiovascular risk. Atherosclerosis. 2019;290:140-205. 
20. Cholesterol Treatment Trialists C, Baigent C, Blackwell L, Emberson J, Holland LE, Reith C, et al. Efficacy and safety of more intensive lowering of LDL cholesterol: a meta-analysis of data from 170,000 participants in 26 randomised trials. Lancet. 2010;376(9753):1670-81.

21. Hofer S, Lim L, Guyatt G, Oldridge N. The MacNew Heart Disease health-related quality of life instrument: a summary. Health Qual Life Outcomes. 2004;2:3.

22. Herdman M, Gudex C, Lloyd A, Janssen M, Kind P, Parkin D, et al. Development and preliminary testing of the new five-level version of EQ-5D (EQ-5D-5L). Qual Life Res. 2011;20(10):1727-36.

23. Osman A, Wong JL, Bagge CL, Freedenthal S, Gutierrez PM, Lozano G. The Depression Anxiety Stress Scales-21 (DASS-21): further examination of dimensions, scale reliability, and correlates. J Clin Psychol. 2012;68(12):1322-38.

24. Hindricks G, Potpara T, Dagres N, Arbelo E, Bax JJ, Blomstrom-Lundqvist C, et al. 2020 ESC Guidelines for the diagnosis and management of atrial fibrillation developed in collaboration with the European Association of Cardio-Thoracic Surgery (EACTS). Eur Heart J. 2020.

25. Piotrowicz R, Krzesinski P, Balsam P, Kempa M, Glowczynska R, Grabowski M, et al. [Cardiology telemedicine solutions - opinion of the experts of the Committee of Informatics and Telemedicine of Polish Society of Cardiology, Section of Non-invasive Electrocardiology and Telemedicine of Polish Society of Cardiology and Clinical Sciences C]. Kardiol Pol. 2018;76(3):698-707.

26. Johnston N, Bodegard J, Jerstrom S, Akesson J, Brorsson H, Alfredsson J, et al. Effects of interactive patient smartphone support app on drug adherence and lifestyle changes in myocardial infarction patients: A randomized study. Am Heart J. 2016;178:85-94.

\section{Tables}

Table 1. Risk factors targets for endpoint assessment

\begin{tabular}{|lll|}
\hline Risk factor & Target level \\
\hline LDL-c level & $\begin{array}{l}\text { No history of Ml } \\
\text { within 2 years prior }\end{array}$ & $\begin{array}{l}\text { LDL-C lowered by at least } 50 \% \text { from baseline and to }<1.4 \\
\mathrm{mmol} / \mathrm{L}(<55 \mathrm{mg} / \mathrm{dL})\end{array}$ \\
\cline { 2 - 3 } & $\begin{array}{l}\text { History of MI within } \\
2 \text { years prior }\end{array}$ & $\begin{array}{l}\mathrm{LDL}-\mathrm{C} \text { of }<1.0 \mathrm{mmol} / \mathrm{L}(<40 \mathrm{mg} / \mathrm{dL}) \text { in patients who have } \\
\text { experience a second vascular event within 2 years }\end{array}$ \\
\hline Body mass index & $18.5-24.9\left(\mathrm{~kg} / \mathrm{m}^{2}\right)$ \\
\hline $\begin{array}{l}\text { Systolic } \\
\text { blood } \\
\text { pressure }\end{array}$ & $\begin{array}{l}\text { General } \\
\text { Older (aged }>65\end{array}$ & $120-130 \mathrm{mmHg}$ \\
\hline Nicotinism & $130-140 \mathrm{mmHg}$ \\
\hline
\end{tabular}

Abbreviations: $\mathrm{MI}=$ Myocardial infarction; LDL= Low-density lipoprotein cholesterol 
Figures

\begin{tabular}{|c|c|c|c|c|}
\hline \multirow[b]{3}{*}{ TIMEPOINT } & \multicolumn{4}{|c|}{ STUDY PERIOD } \\
\hline & \multirow{2}{*}{$\begin{array}{c}\begin{array}{c}\text { Enrolme } \\
\text { nt }\end{array} \\
-t_{1}\end{array}$} & \multirow{2}{*}{$\begin{array}{c}\begin{array}{c}\text { Allocatio } \\
\mathbf{n}\end{array} \\
\mathbf{0} \\
\text { (during } \\
\text { hospitalizatio } \\
\text { ndue to MI) } \\
\end{array}$} & \multicolumn{2}{|c|}{ Post-allocation } \\
\hline & & & $\begin{array}{c}t_{1} \\
\text { (30 days after discharge } \\
\text { from hospital) }\end{array}$ & $\begin{array}{c}t_{2} \\
\text { (6 months after discharge } \\
\text { from hospital) }\end{array}$ \\
\hline \multirow{4}{*}{$\begin{array}{r}\text { ENROLMENT: } \\
\text { Eligibility } \\
\text { screen } \\
\text { Informed } \\
\text { consent } \\
\text { Allocation }\end{array}$} & & & & \\
\hline & $x$ & & & \\
\hline & $x$ & & & \\
\hline & & $x$ & & \\
\hline \multicolumn{5}{|l|}{ INTERVENTION } \\
\hline \\
\hline \multicolumn{5}{|l|}{ Control Group } \\
\hline \multicolumn{5}{|l|}{ ASSESSMENTS } \\
\hline \multirow{2}{*}{$\begin{array}{c}\text { Laboratory test } \\
\text { Rehospitalizati } \\
\text { on or urgent } \\
\text { outpatients } \\
\text { visits }\end{array}$} & & $\mathrm{x}$ & & \\
\hline & & & $x$ & $\mathrm{x}$ \\
\hline \multirow{6}{*}{$\begin{array}{r}\text { Cardiovascular } \\
\text { risk factors } \\
\text { control } \\
\text { Quality of life - } \\
\text { MacNew } \\
\text { Quality of life- } \\
\text { EQ-5D-5L } \\
\text { Depression- } \\
\text { DASS21 } \\
\text { Cardiovascular } \\
\text { risk factors } \\
\text { knowledge }\end{array}$} & & & $x$ & $x$ \\
\hline & & $x$ & $\mathrm{x}$ & $x$ \\
\hline & & $\mathrm{x}$ & $\mathrm{x}$ & $\mathrm{x}$ \\
\hline & & $x$ & $x$ & $x$ \\
\hline & & $\mathrm{x}$ & $\mathrm{x}$ & $\mathrm{x}$ \\
\hline & & & $\mathrm{x}$ & $x$ \\
\hline
\end{tabular}

Figure 1

Recommended SPIRIT figure with participant timeline. 


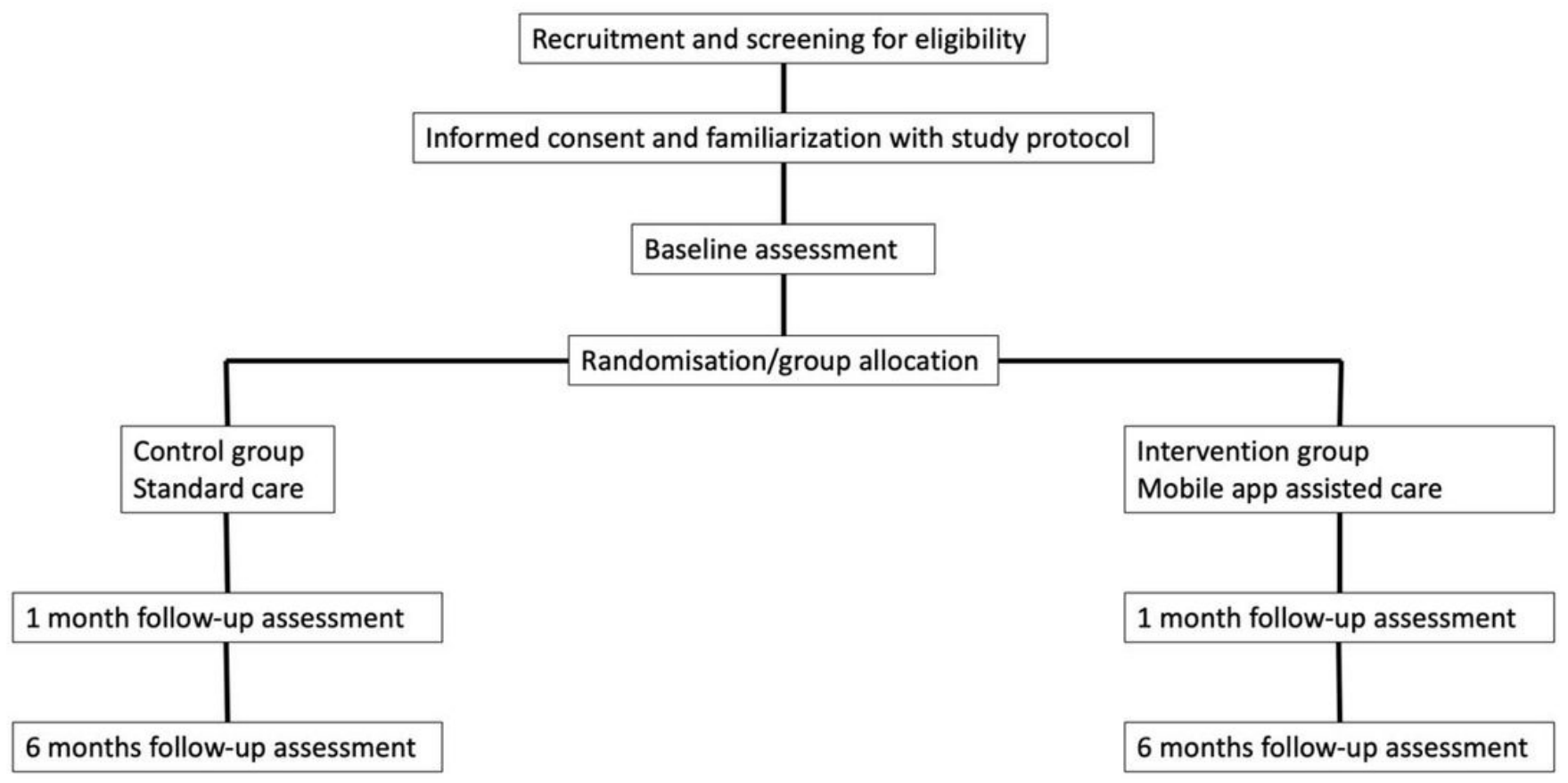

Figure 2

Study flow chart.

\section{Supplementary Files}

This is a list of supplementary files associated with this preprint. Click to download.

- CONSORTafterAMI.doc 